\title{
An A/C germline single-nucleotide polymorphism in the TNFAIP3 gene is associated with advanced disease stage and survival in only surgically treated esophageal cancer
}

Tarik Ghadban, Magdalena Schmidt-Yang, Faik G Uzunoglu, Daniel R Perez, Tung Y Tsui, Alexander T El Gammal, Peter J Erbes, Veacheslav Zilbermints, Ulrich Wellner, Klaus Pantel, Jakob R Izbicki and Yogesh K Vashist

Journal of Human Genetics (2015) 60, 107; doi:10.1038/jhg.2014.106

Correction to: Journal of Human Genetics advance online publication, 30 October 2014; doi:10.1038/jhg.2014.90

The authors of the above article noticed an error in publication of this article (in December 2014 issue).
In the list of authors, last name of Magdalena Schmidt-Yang was wrong. The correct name is now shown above.

The authors apologize for any inconvenience caused. 